\title{
Bifurcation Analysis in a Two-Dimensional Neutral Differential Equation
}

\author{
Ming Liu and Xiaofeng Xu \\ Department of Mathematics, Northeast Forestry University, Harbin 150040, China \\ Correspondence should be addressed to Xiaofeng Xu; xxf_ray@163.com
}

Received 2 February 2013; Accepted 5 April 2013

Academic Editor: Chunrui Zhang

Copyright (C) 2013 M. Liu and X. Xu. This is an open access article distributed under the Creative Commons Attribution License, which permits unrestricted use, distribution, and reproduction in any medium, provided the original work is properly cited.

\begin{abstract}
The dynamics of a 2-dimensional neural network model in neutral form are investigated. We prove that a sequence of Hopf bifurcations occurs at the origin as the delay increases. The direction of the Hopf bifurcations and the stability of the bifurcating periodic solutions are determined by using normal form method and center manifold theory. Global existence of periodic solutions is established using a global Hopf bifurcation result of Krawcewicz et al. Finally, some numerical simulations are carried out to support the analytic results.
\end{abstract}

\section{Introduction}

The fundamental theories and applications of neutral functional differential equations have been largely developed and there are a great deal of important conclusions on the stability, bifurcation theory, and numerical solutions of neutral delay differential equations during the last few decades. It is well known that Wei and Ruan [1] have established a basic theory to analyze the distribution of the zeros of general transcendental equations and applied it to the neutral functional differential equations. One can use the theory to study the existence of Hopf bifurcation to a neutral delay differential equation. In [2], Wang and Wei extend the computation of the properties of Hopf bifurcation, such as the direction of bifurcation and stability of bifurcating periodic solutions, of DDE introduced by Kazarinoff et al. Based on combining the global Hopf bifurcation theory of neutral equations due to Krawcewicz et al. and the higher dimensional Bendixson's criterion for ordinary differential equations due to Li and Muldowney, the global existence of periodic solutions of neutral differential equations have been studied by $\mathrm{Qu}$ et al. [3]. But research findings of bifurcation about highdimension neutral differential equations can rarely be found.

In order to determine the dynamics of artificial neural network, the two-dimensional delay neural network without self-feedback is studied, and some significant results are obtained (see [4-8]). In this paper, we consider the following system:

$$
\begin{aligned}
& \dot{x}_{1}(t)=-\mu x_{1}(t)+a f\left(x_{1}(t-\tau)\right)+b \dot{x}_{2}(t-\tau), \\
& \dot{x}_{2}(t)=-\mu x_{2}(t)+a f\left(x_{2}(t-\tau)\right)+b \dot{x}_{1}(t-\tau),
\end{aligned}
$$

where $\mu, a$, and $b$ are real numbers with $\mu>0$ and $|b|<1$. $f$ is assumed adequately smooth, for example, $f \in C^{3}$, and satisfies the following condition:

(H1) $f(0)=0, f^{\prime}(0)=1$, and there exists $L>0$, such that $|f(x)| \leqslant L$ for all $x \in \mathbb{R}$,

(H2) $f^{\prime}(x)>0$ for all $x \in \mathbb{R}, x f^{\prime \prime}(x)<0$ for all $x \neq 0$.

In the present paper, we provide a detailed analysis of this equation. By applying the local Hopf bifurcation theory (see [9] and also $[1,2,10,11]$ ), we investigate the existence of stable periodic oscillations for (1). More specially, we prove that the equilibrium $(0,0)$ loses its stability as $\tau$ increases, and a sequence of Hopf bifurcations occurs at the origin. Whereafter, based on the normal form and center manifold theory due to $[12,13]$, by using the method introduced in Wang and Wei [2], we derive a sufficient condition for determining the direction of Hopf bifurcation and the stability of the bifurcating periodic solutions. Furthermore, 
the existence of periodic solutions for $\tau$ far away from the Hopf bifurcation values is also established, by using a global Hopf bifurcation result due to Krawcewicz et al. [14] (also see $[15,16])$.

The rest of this paper is organized as follows: in Section 2, taking $a$ and $\tau$ as parameters, we give the analysis of stability and bifurcations at equilibria. Section 3 is devoted to establishing the direction and stability of Hopf bifurcation. Finally, a global Hopf bifurcation is established, and some numerical simulations are carried out to illustrate the analytic results in Section 4.

\section{Stability and Bifurcation Analysis}

In this section, we will investigate the stability and bifurcations of the equilibria by taking $a$ and $\tau$ as bifurcation parameters.

Under the given hypothesis (H1), it is easy to check that $(0,0)$ is an equilibrium point of system (1). At this trivial equilibrium, the linearization of system (1) is

$$
\dot{X}(t)=A X(t)+B X(t-\tau)+C \dot{X}(t-\tau),
$$

where

$$
A=\left(\begin{array}{cc}
-\mu & 0 \\
0 & -\mu
\end{array}\right), \quad B=\left(\begin{array}{cc}
a & 0 \\
0 & a
\end{array}\right), \quad C=\left(\begin{array}{ll}
0 & b \\
b & 0
\end{array}\right) .
$$

And the corresponding characteristic equation is

$$
\Delta(\lambda)=\left(\lambda+\mu-a e^{-\lambda \tau}\right)^{2}-b^{2} \lambda^{2} e^{-2 \lambda \tau}=0,
$$

that is,

$$
\begin{gathered}
\left(\lambda+\mu-(a-b \lambda) e^{-\lambda \tau}\right)\left(\lambda+\mu-(a+b \lambda) e^{-\lambda \tau}\right) \\
\stackrel{\text { def }}{=} \Delta_{1}(\lambda) \cdot \Delta_{2}(\lambda)=0 .
\end{gathered}
$$

Case 1. Choose $a$ as parameter.

For convenience, we give two claims at first.

Claim 1. All the roots of (4) with $a=0$ have negative real parts for any $\tau \geqslant 0$.

Proof. When $a=0$ and $\tau=0$, (4) becomes $(\lambda+\mu)^{2}-b^{2} \lambda^{2}=0$, and the roots are $\lambda_{1,2}=-\mu /(1 \pm b)<0$. solves

$i v_{0}\left(v_{0}>0\right)$ is a zero of $\Delta(\lambda)$ with $a=0$ if and only if $v_{0}$

$$
\left(i v_{0}+\mu\right)^{2}+b^{2} v_{0}^{2}\left(\cos 2 v_{0} \tau-i \sin 2 v_{0} \tau\right)=0
$$

Separating the real and imaginary parts gives that $\left(b^{2}-1\right) v_{0}^{2}=$ $\mu^{2}$, which contradicts with $|b|<1$. Then the conclusion follows Wei and Ruan [1], and the proof is complete.

Claim 2. Equation (4) has at least two positive roots when $a>\mu$.

In fact, the conclusion follows from $\Delta_{1,2}(0)=\mu-a<0$ and $\lim _{\lambda \rightarrow+\infty} \Delta_{1,2}(\lambda)=+\infty$.
When $b \neq 0$, iv $(v>0)$ is a root of (4) if and only if $v$ satisfies

$$
\begin{gathered}
a \sin v \tau=-v(1+b \cos v \tau) \\
a \cos v \tau=u+b v \sin v \tau
\end{gathered}
$$

or

$$
\begin{gathered}
a \sin v \tau=-v(1-b \cos v \tau), \\
a \cos v \tau=u-b v \sin v \tau .
\end{gathered}
$$

Applying the method in [3], we have that there exists a unique value of $v$, denoted by $v_{1 m}^{+}\left(v_{1 m}^{-}\right)$, satisfying $(7)$ and a unique value of $v$, denoted by $v_{2 m}^{+}\left(v_{2 m}^{-}\right)$, satisfying (8) on the interval $(2(m-1) \pi / \tau, 2 m \pi / \tau), m \in \mathbb{Z}^{+}$when $a>0(<$ $0)$. We also can show that $v_{k m}^{+} \in((2 m-1) \pi / \tau, 2 m \pi / \tau), k=$ $1,2, m \in \mathbb{Z}^{+}$with $a>0$ and $v_{k m}^{-} \in(2(m-1) \pi / \tau,(2 m-$ 1) $\pi / \tau), k=1,2, m \in \mathbb{Z}^{+}$with $a<0$.

Define

$$
a_{k m}^{ \pm}= \pm \sqrt{v_{k m}^{ \pm}{ }^{2}\left(1-b^{2}\right)+\mu^{2}}, \quad k=1,2, m \in \mathbb{Z}^{+} .
$$

$a_{k m}^{ \pm}$make sense since $|b|<1$, and $a_{k m}^{+}$and $a_{k m}^{-}$are all dependent on $\tau$. Hence, $\pm v_{k m}^{+}\left( \pm v_{k m}^{-}\right)$are a pair of purely imaginary roots of (4) with $a=a_{k m}^{+}\left(a_{k m}^{-}\right)$. And we have the transversal condition

$$
\begin{aligned}
\left.\operatorname{Sign} \operatorname{Re}\left(\frac{d \lambda}{d a}\right)\right|_{a=a_{k m}^{ \pm}} \\
=\left\{\begin{array}{ll}
1, & a=a_{k m}^{+}, \\
-1, & a=a_{k m}^{-},
\end{array} \quad k=1,2, m \in \mathbb{Z}^{+} .\right.
\end{aligned}
$$

Moreover, it is fulfilled that $a_{k \times(m+1)}^{-}<a_{k m}^{-}<0<a_{k m}^{+}<$ $a_{k \times(m+1)}^{+}$, following the fact that $0<v_{k m}^{-}<v_{k m}^{+}<v_{k \times(m+1)}^{-}<$ $v_{k \times(m+1)}^{+}$. Now we can distinguish two cases. First, $b>0$. From (7) and (8), we can obtain that $a_{1 m}^{-} \cos v_{1 m}^{-} \tau>a_{2 m}^{-} \cos v_{2 m}^{-} \tau$, which implies $v_{1 m}^{-}>v_{2 m}^{-}$and $a_{1 m}^{-}<a_{2 m}^{-}$. Second, $b<0$. With the similar process as above, it is obtained that $v_{1 m}^{-}<v_{2 m}^{-}$and $a_{1 m}^{-}>a_{2 m}^{-}$.

Summarizing the discussion above and applying Claims 1 and 2, we have the following.

Lemma 1. There exist sequence values of a defined by (9) such that all the roots of (4) have negative real parts when $a \in\left(a_{1}^{-}(\tau), \mu\right)$, and (4) has at least two roots with positive real part when $a \in\left(-\infty, a_{1}^{-}(\tau)\right) \cup(\mu,+\infty)$, a pair of purely imaginary roots when $a=a_{k m}^{ \pm}(\tau)\left(k=1,2, m \in \mathbb{Z}^{+}\right)$, where $a_{1}^{-}(\tau)=a_{11}^{-}\left(a_{21}^{-}\right)$when $b<0(>0)$.

Lemma 2. Equation (1) undergoes a pitchfork bifurcation at $(0,0)$ when $a=\mu$.

Proof. Let $h(x)=-\mu x+a f(x)$. Then we have $h(0)=0$, $h( \pm \infty)=\mp \infty$, and $h^{\prime}(x)=-\mu+a f^{\prime}(x)$. From (H1) and (H2), there exists a unique zero $x=0$ of $h(x)$, and (1) has only one steady-state $X=(0,0)$ when $a \leq \mu$. On the other hand, when $a>\mu$, we have $h^{\prime}(0)=a-\mu>0$ and $x h^{\prime \prime}(x)<0$ when $x \neq 0$. Thus, there exists a unique $c^{+} \in \mathbb{R}^{+}\left(c^{-} \in \mathbb{R}^{-}\right)$satisfying $h(x)=0$, and (1) has exactly nonzero equilibria $\left(c^{ \pm}, 0\right),\left(0, c^{ \pm}\right)$, and $\left(c^{ \pm}, c^{ \pm}\right)$. This completes the proof. 
In the following, we will investigate the stability of $\left(c^{ \pm}, 0\right)$, $\left(0, c^{ \pm}\right)$, and $\left(c^{ \pm}, c^{ \pm}\right)$.

Firstly, we consider the stability of $\left(c^{+}, 0\right)$ when $a>\mu$. (It has similar results with $\left(c^{-}, 0\right)$ and $\left(0, c^{ \pm}\right)$.) is

The characteristic equation of linearization of $(1)$ at $\left(c^{+}, 0\right)$

$$
\begin{aligned}
\Delta_{\left(c^{+}, 0\right)}(\lambda)= & \left(\lambda+\mu-a e^{-\lambda \tau}\right)\left(\lambda+\mu-a f^{\prime}\left(c^{+}\right) e^{-\lambda \tau}\right) \\
& -b^{2} \lambda^{2} e^{-2 \lambda \tau}=0
\end{aligned}
$$

Equation (11) has at least one positive root for any $\tau \geq 0$. In fact, we have $h^{\prime}\left(c^{ \pm}\right)<0$ and $0<a f^{\prime}\left(c^{ \pm}\right)<\mu$ from the proof of Lemma 2. Then the conclusion follows from $\Delta_{\left(c^{+}, 0\right)}(0)=$ $(\mu-a)\left(\mu-a f^{\prime}\left(c^{+}\right)\right)<0$ and $\lim _{\lambda \rightarrow+\infty} \Delta_{\left(c^{+}, 0\right)}(\lambda)=+\infty$, which implies that $\left(c^{+}, 0\right)$ is unstable.

Secondly, we consider the stability of $\left(c^{+}, c^{+}\right)$when $a>\mu$. (It has similar results with $\left(c^{+}, c^{-}\right)$and $\left(c^{-}, c^{ \pm}\right)$).

The characteristic equation of linearization of (1) at $\left(c^{+}, c^{+}\right)$is

$$
\begin{aligned}
\Delta_{\left(c^{+}, c^{+}\right)}(\lambda, \tau)= & \left(\lambda+\mu-a f^{\prime}\left(c^{+}\right) e^{-\lambda \tau}\right)^{2} \\
& -b^{2} \lambda^{2} e^{-2 \lambda \tau}=0 .
\end{aligned}
$$

In particular,

$$
\Delta_{\left(c^{+}, c^{+}\right)}(\lambda, 0)=\left(\lambda+\mu-a f^{\prime}\left(c^{+}\right)\right)^{2}-b^{2} \lambda^{2}=0,
$$

which implies

$$
\lambda_{1,2}=\frac{-\mu+a f^{\prime}\left(c^{+}\right)}{1 \pm b}<0 .
$$

Moreover, (12) has no zero root from $a f^{\prime}\left(c^{+}\right)<\mu$.

In order to prove that $\left(c^{+}, c^{+}\right)$is asymptotically stable for all $\tau \in \mathbb{R}^{+}$, what we need is to verify that (12) has no purely imaginary roots. Let $i \eta(\eta>0)$ be the root of $(12)$, then $\eta$ solves

$$
\begin{gathered}
a f^{\prime}\left(c^{+}\right) \sin \eta \tau+b \eta \cos \eta \tau=-\eta \\
a f^{\prime}\left(c^{+}\right) \cos \eta \tau-b \eta \sin \eta \tau=\mu
\end{gathered}
$$

or

$$
\begin{aligned}
& a f^{\prime}\left(c^{+}\right) \sin \eta \tau-b \eta \cos \eta \tau=-\eta, \\
& a f^{\prime}\left(c^{+}\right) \cos \eta \tau+b \eta \sin \eta \tau=\mu .
\end{aligned}
$$

This leads to

$$
\eta^{2}=\frac{\left(a f^{\prime}\left(c^{+}\right)\right)^{2}-\mu^{2}}{1-b^{2}}<0 .
$$

The assertion follows.

Applying Lemmas 1 and 2, we have the following results.

Theorem 3. (i) The zero solution of (1) is asymptotically stable when $a \in\left(a_{1}^{-}(\tau), \mu\right)$ and unstable when $a \in\left(-\infty, a_{1}^{-}(\tau)\right) \cup$ $(\mu,+\infty)$. (ii) Equation (1) undergoes a pitchfork bifurcation at $(0,0)$ when $a=\mu$. More precisely, some new equilibria bifurcate from zero and $\left(c^{ \pm}, 0\right),\left(0, c^{ \pm}\right)$are unstable, and $\left(c^{ \pm}, c^{ \pm}\right)$are asymptotically stable for $\tau \in \mathbb{R}^{+}$when $a>\mu$.

(iii) Equation (1) undergoes a Hopf bifurcation at $(0,0)$ when $a=a_{k m}^{ \pm}(\tau)\left(k=1,2, m \in \mathbb{Z}^{+}\right)$.

Case 2. Regard $\tau$ as parameter.

First of all, we know that the root of (4) with $\tau=0$ satisfies that $\lambda_{1,2}=(a-\mu) /(1 \pm b)>0$ when $a>\mu$, and $\lambda_{1,2}=$ $(a-\mu) /(1 \pm b)<0$ when $a<\mu$.

Let $i \omega(\omega>0)$ be a root of $(4)$; then it follows that

$$
\sin \omega \tau=-\frac{\omega(a \pm b \mu)}{\mu^{2}+\omega^{2}}, \quad \cos \omega \tau=\frac{a \mu \mp b \omega^{2}}{\mu^{2}+\omega^{2}} .
$$

This leads to $\omega^{2}=\left(a^{2}-\mu^{2}\right) /\left(1-b^{2}\right), \omega_{0} \stackrel{\text { def }}{=} \sqrt{\left(a^{2}-\mu^{2}\right) /\left(1-b^{2}\right)}$ makes sense when $|a|>\mu$. Define

$$
\begin{aligned}
& \tau_{1 j} \\
& =\left\{\begin{array}{ll}
\frac{1}{\omega_{0}}\left(\arccos \frac{a \mu-b \omega_{0}^{2}}{\mu^{2}+\omega_{0}^{2}}+2 j \pi\right), & a<-\mu, \\
\frac{1}{\omega_{0}}\left(-\arccos \frac{a \mu-b \omega_{0}^{2}}{\mu^{2}+\omega_{0}^{2}}+2(j+1) \pi\right), & a>\mu,
\end{array} \quad j=0,1,2, \ldots,\right. \\
& \tau_{2 j} \\
& =\left\{\begin{array}{ll}
\frac{1}{\omega_{0}}\left(\arccos \frac{a \mu+b \omega_{0}^{2}}{\mu^{2}+\omega_{0}^{2}}+2 j \pi\right), & a<-\mu, \\
\frac{1}{\omega_{0}}\left(-\arccos \frac{a \mu+b \omega_{0}^{2}}{\mu^{2}+\omega_{0}^{2}}+2(j+1) \pi\right), & a>\mu,
\end{array} \quad j=0,1,2, \ldots .\right.
\end{aligned}
$$

Then $i \omega_{0}$ is a purely imaginary root of (4) with $\tau=\tau_{k j}, k=$ 1,2 defined by (19).

Let $\lambda(\tau)=\alpha(\tau)+i \varrho(\tau)$ be the root of (4), satisfying

$$
\alpha\left(\tau_{k j}\right)=0, \quad \varrho\left(\tau_{k j}\right)=\omega_{0} .
$$

Differentiating both side of (4) gives that

$$
\left.\operatorname{Re}\left(\frac{d \lambda}{d \tau}\right)^{-1}\right|_{\tau=\tau_{k j}}=\frac{1-b^{2}}{a^{2}+b^{2} \omega_{0}^{2}}>0
$$

This implies that $\alpha^{\prime}\left(\tau_{k j}\right)>0, k=1,2, j=0,1,2, \ldots$

Summarizing the discussions above, one can obtain the following.

Lemma 4. (i) If $|a|<\mu$, then all roots of (4) have negative real parts.

(ii) If $|a|>\mu$, then there exist a sequence values of $\tau$ defined by (19) such that (4) has a pair of purely imaginary roots $\pm i \omega_{0}$ when $\tau=\tau_{k j}, k=1,2$. Additionally, if $a<-\mu$, then all roots of (4) have negative real parts when $\tau \in\left[0, \tau_{0}\right)$, all roots of (4), except $\pm i \omega_{0}$, have negative real parts when $\tau=\tau_{0}$, and (4) has at least a pair of root with positive real parts when $\tau>\tau_{0}$, where $\tau_{0}=\tau_{10}\left(\tau_{20}\right)$ when $b<0(>0)$; if $a>\mu$, then (4) has at least two positive roots. 
Spectral properties in Lemma 4 immediately lead to the dynamics near the origin described by the following theorem.

Theorem 5. For (1), the following holds.

(i) If $|a|<\mu$, then $(0,0)$ is asymptotically stable for all $\tau>0$.

(ii) If $a<-\mu$, then $(0,0)$ is asymptotically stable when $\tau \in$ $\left(0, \tau_{0}\right)$, and unstable when $\tau>\tau_{0}$.

(iii) If $a>\mu$, then $(0,0)$ is always unstable for all $\tau>0$.

(iv) If $|a|>\mu$, then (1) undergoes a Hopf bifurcation at $(0,0)$ when $\tau=\tau_{k j}, k=1,2, j=0,1,2, \ldots$.

\section{Properties of Hopf BifurCation}

Theorems 3(iii) and 5(iv) in the previous section give the sufficient conditions for (1) to undergo Hopf bifurcations with $a$ and $\tau$ as bifurcation parameters. In this section, we will investigate the direction of Hopf bifurcations and stability of bifurcating periodic solutions, following the same algorithms as Wang and Wei's recent work and using a computation process similar to that in [2] (see also [17]). We should mention that we will choose $\tau$ as bifurcation parameter, and the similar results follow when choosing other coefficients as bifurcation parameters.

Let $y_{i}(t)=x_{i}(\tau t), i=1,2$, and $Y(t)=\left(y_{1}(t), y_{2}(t)\right)^{T}$, then (1) becomes

$$
\frac{d}{d t}[Y(t)-C Y(t-1)]=\tau A Y(t)+\tau B F(Y(t-1)),
$$

where $A, B$, and $C$ are denoted by (2) and $F(Y(t-1))=$ $\left(f\left(y_{1}(t-1)\right), f\left(y_{2}(t-1)\right)\right)^{T}$. And the corresponding characteristic equation around $Y=(0,0)^{T}$ is

$$
\left(z+\mu \tau-a \tau e^{-z}\right)^{2}-b^{2} z^{2} e^{-2 z}=0,
$$

Comparing (23) with (4), if is found that $z=\lambda \tau$ for $\tau \neq 0$. Therefore, combining this fact with Lemma 4, one has the following.

\section{Lemma 6. Assume $|a|>\mu$.}

(i) If $\tau=\tau_{j}, j=0,1,2, \ldots$, then (23) has a pair of purely imaginary roots $\pm i \omega_{0} \tau_{j}$, where $\tau_{j}=\tau_{k j}$ and $\omega_{0}$ are defined by (19).

(ii) Let $z(\tau)=\gamma(\tau)+i \varsigma(\tau)$ be the root of (23), satisfying

$$
\gamma\left(\tau_{j}\right)=0, \quad \varsigma\left(\tau_{j}\right)=\omega_{0} \tau_{j},
$$

then

$$
\gamma^{\prime}\left(\tau_{j}\right)=\tau_{j} \alpha^{\prime}\left(\tau_{j}\right)>0, \quad j=0,1,2, \ldots
$$

(iii) Equation (23) has at least a pair of roots with positive real parts when $\tau=\tau_{j}$ for $j \geq 1$, and it has two positive roots when $a>\mu$ and $\tau=\tau_{0}$. All roots of (23) with $\tau=\tau_{0}$, except $\pm i \omega_{0} \tau_{0}$, have negative real parts when $a<-\mu$.
For convenience, denote $\tau=\tau_{j}+\nu$. Then we know that (22) undergoes a Hopf bifurcation at origin when $v=0$. For $\phi \in C\left([-1,0], \mathbb{R}^{2}\right)$, let

$$
\begin{gathered}
D(\phi)=\phi(0)-C \phi(-1), \\
L(\nu, \phi)=A\left(\tau_{j}+\nu\right) \phi(0)+B\left(\tau_{j}+\nu\right) \phi(-1), \\
F(\nu, \phi)=\frac{f^{\prime \prime \prime}(0)}{6}\left(\tau_{j}+\nu\right) B \phi^{3}(-1)+\cdots .
\end{gathered}
$$

By the Riesz representation theorem, there exist functions $\eta(\theta)$ and $\mu(\theta)$ such that

$$
\begin{gathered}
D(\phi)=\phi(0)-\int_{-1}^{0} d \mu(\theta) \phi(\theta), \\
L(\nu, \phi)=\int_{-1}^{0} d \eta(\theta) \phi(\theta) .
\end{gathered}
$$

In fact, we can choose

$$
\begin{gathered}
\mu(\theta)= \begin{cases}-C, & \theta=-1, \\
0, & \theta \in(-1,0],\end{cases} \\
\eta(\theta)= \begin{cases}-B\left(\tau_{j}+v\right), & \theta=-1, \\
0, & \theta \in(-1,0), \\
A\left(\tau_{j}+v\right), & \theta=0 .\end{cases}
\end{gathered}
$$

Define

$$
\begin{gathered}
A(\nu) \phi= \begin{cases}\frac{d \phi(\theta)}{d \theta}, & \theta \in[-1,0), \\
\phi^{\prime}(0)-D \phi^{\prime}(0)+L \phi, & \theta=0,\end{cases} \\
R(\nu) \phi= \begin{cases}0, & \theta \in[-1,0), \\
F(\nu, \phi), & \theta=0 .\end{cases}
\end{gathered}
$$

Then (22) can be written as

$$
\dot{Y}_{t}=A(\nu) Y_{t}+R(\nu) Y_{t}
$$

Clearly, (30) is an abstract ODE on the phase space $B C^{\prime}$ of (22), where $B C^{\prime}=\left\{\phi:[-1,0] \rightarrow \mathbb{C}^{2}, \phi\right.$ is continuous on $[-1,0)$, and $\lim _{\theta \rightarrow 0} \phi(\theta)$ exists $\}$.

The adjoint operator $A^{*} \psi=-d \psi / d s$ with domain

$$
\begin{aligned}
D & \left(A^{*}\right) \\
& =\left\{\psi \in C^{\prime} \stackrel{\text { def }}{=} C\left([0,1], \mathbb{R}^{2}\right): \frac{d \psi}{d s} \in C^{\prime} ; D \frac{d \psi}{d s}=-L \psi\right\} .
\end{aligned}
$$

For $(\psi, \phi) \in C^{\prime} \times C$, define a bilinear form:

$$
\begin{aligned}
(\psi, \phi)= & \bar{\psi}(0) \phi(0) \\
& -\int_{-1}^{0} d\left[\int_{\alpha=0}^{\theta} \bar{\psi}(\theta-\alpha) d \mu(\alpha)\right] \phi(\theta) \\
& -\int_{-1}^{0} \int_{\theta=0}^{s} \bar{\psi}(\theta-s) d \eta(s) \phi(\theta) d \theta
\end{aligned}
$$


It is not difficult to verify that $q(\theta)=q_{0} e^{i \omega_{0} \tau_{j} \theta}(\theta \in[-1,0])$ and $q^{*}(s)=\bar{l} q_{0}^{*} e^{i \omega_{0} \tau_{j} s}(s \in[0,1])$ are the eigenvectors of $A(0)$ and $A^{*}$ corresponding to the eigenvalues $i \omega_{0} \tau_{j}$ and $-i \omega_{0} \tau_{j}$, respectively, where

$$
\begin{gathered}
q_{0}= \begin{cases}(1,-1)^{T} & \tau_{j}=\tau_{1 j}, \\
(1,1)^{T} & \tau_{j}=\tau_{2 j},\end{cases} \\
q_{0}^{*}= \begin{cases}(1,-1) & \tau_{j}=\tau_{1 j}, \\
(1,1) & \tau_{j}=\tau_{2 j},\end{cases} \\
l=\frac{1}{q_{0}^{*}\left(I-C e^{-i \omega_{0} \tau_{j}}+B \tau_{j} e^{-i \omega_{0} \tau_{j}}\right) \bar{q}_{0}},
\end{gathered}
$$

and $\left(q^{*}, q\right)=1$.
Now we compute the center manifold $C_{0}$ at $\nu=0$. Define

$$
\begin{gathered}
z(t)=\left(q^{*}, Y_{t}\right), \\
W(t, \theta)=Y_{t}(\theta)-2 \operatorname{Re} z(t) q(\theta),
\end{gathered}
$$

then we have

$$
\dot{z}(t)=i \omega_{0} \tau_{j} z+\bar{q}^{*}(0) F\left(0, Y_{t}\right) .
$$

Equation (35) can be written in the abbreviated form as

$$
\dot{z}(t)=i \omega_{0} \tau_{j} z+g(z, \bar{z}),
$$

with

$$
g(z, \bar{z})=g_{20} \frac{z^{2}}{2}+g_{11} z \bar{z}+g_{02} \frac{\bar{z}^{2}}{2}+g_{21} \frac{z^{2} \bar{z}}{2}+\cdots .
$$

Noting that $Y_{t}(\theta)=W(t, \theta)+z(t) q(\theta)+\bar{z}(t) \bar{q}(\theta)$, we have

$$
Y_{t}(-1)=\left(\begin{array}{l}
q_{01} e^{-i \omega_{0} \tau_{j}} z+\bar{q}_{01} e^{i \omega_{0} \tau_{j}} \bar{z}+W_{20}^{(1)}(-1) \frac{z^{2}}{2}+W_{11}^{(1)}(-1) z \bar{z}+W_{02}^{(1)}(-1) \frac{\bar{z}^{2}}{2}+\cdots \\
q_{02} e^{-i \omega_{0} \tau_{j}} z+\bar{q}_{02} e^{i \omega_{0} \tau_{j}} \bar{z}+W_{20}^{(2)}(-1) \frac{z^{2}}{2}+W_{11}^{(2)}(-1) z \bar{z}+W_{02}^{(2)}(-1) \frac{\bar{z}^{2}}{2}+\cdots
\end{array}\right),
$$

where $q_{0}=\left(q_{01}, q_{02}\right)^{T}$. Therefore, from (26), we have

$$
\begin{aligned}
F\left(0, Y_{t}\right) & =\frac{f^{\prime \prime \prime}(0)}{6} \tau_{j} Y_{t}^{3}(-1)+\cdots \\
& \stackrel{\operatorname{def}}{=} F_{z^{2}} \frac{z^{2}}{2}+F_{z \bar{z}} z \bar{z}+F_{\bar{z}^{2}} \frac{\bar{z}^{2}}{2}+F_{z^{2} \bar{z}} \frac{z^{2} \bar{z}}{2}+\cdots
\end{aligned}
$$

Substituting the expression of $F\left(0, Y_{t}\right)$ into (35) and comparing its coefficients with that of (36) give that

$$
\begin{gathered}
g_{20}=g_{02}=g_{11}=0 \\
g_{21}=\bar{l} \bar{q}_{0}^{*} \frac{f^{\prime \prime \prime}(0)}{6} \tau_{j} B\left(\begin{array}{l}
q_{01}^{2} \bar{q}_{01} \\
q_{02}^{2} \bar{q}_{02}
\end{array}\right) e^{-i \omega_{0} \tau_{j}} .
\end{gathered}
$$

It is well known that the coefficient $c_{1}(0)$ of third degree term of Poincaré normal form of (35) is given by (see [12])

$$
c_{1}(0)=\frac{i}{2 \omega_{0} \tau_{j}}\left(g_{20} g_{11}-2\left\|g_{11}\right\|^{2}-\frac{1}{3}\left\|g_{02}\right\|^{2}\right)+\frac{g_{21}}{2} .
$$

Consequently, when $\tau=\tau_{1 j}$ or $\tau=\tau_{2 j}$,

$$
\begin{aligned}
c_{1}(0)= & \frac{\tau_{k j}}{12\left\|1+\left(\mp b+a \tau_{k j}\right) e^{-i \omega_{0} \tau_{k j}}\right\|^{2}} \\
& \times a f^{\prime \prime \prime}(0)\left[1+\left(\mp b+a \tau_{k j}\right) e^{-i \omega_{0} \tau_{k j}}\right] e^{-i \omega_{0} \tau_{k j}} .
\end{aligned}
$$

\section{Hence we obtain}

$$
\begin{aligned}
& \text { Sign Rec } c_{1}(0) \\
& =\operatorname{Sign} a f^{\prime \prime \prime}(0) \\
& \quad \times\left[\frac{a \mu \mp b \omega_{0}^{2}}{\mu^{2}+\omega_{0}^{2}}+\left(\mp b+a \tau_{k j}\right)\left(2\left(\frac{a \mu \mp b \omega_{0}^{2}}{\mu^{2}+\omega_{0}^{2}}\right)^{2}-1\right)\right] .
\end{aligned}
$$

Summarizing the above analysis, we have the following theorem.

Theorem 7. For (22), the direction of Hopf bifurcation at $\tau=$ $\tau_{k j}, k=1,2, j=0,1,2, \ldots$, is supercritical (subcritical), and the bifurcating periodic solutions are asymptotically stable (unstable) when $\operatorname{Re} c_{1}(0)<0\left(\operatorname{Re} c_{1}(0)>0\right)$.

\section{Global Hopf Bifurcation Analysis}

Our objective in this section is to obtain the global continuation of periodic solution bifurcating from the point $\left(\mathbf{0}, \tau_{k j}\right), k=1,2, j=0,1,2, \ldots$, for (1) by using a global Hopf bifurcation theorem given by Krawcewicz et al. [14]. For the reader's convenience, we copy (22), which is equivalent to (1), as follows

$$
\begin{aligned}
& \dot{y}_{1}(t)-b \dot{y}_{2}(t-1)=-\mu \tau y_{1}(t)+a \tau f\left(y_{1}(t-1)\right), \\
& \dot{y}_{2}(t)-b \dot{y}_{1}(t-1)=-\mu \tau y_{2}(t)+a \tau f\left(y_{2}(t-1)\right) .
\end{aligned}
$$

We have known that (44) undergoes a local Hopf bifurcation at the origin when $\tau=\tau_{k j}, k=1,2, j=0,1,2, \ldots$. Now we 
begin to show that each bifurcation branch can be continued with respect to the parameter $\tau$ under certain conditions. To bring out the ideas in the results of subsequent part, it is convenient to introduce the following notations:

$$
\begin{aligned}
& X=C\left([-1,0], \mathbb{R}^{2}\right), \\
& \Sigma=C l\{(Y, \tau, T): Y \text { is a } T \text {-periodic solution of }(44)\} \subset \\
& X \times \mathbb{R}_{+} \times \mathbb{R}_{+}, \\
& N=\{(\widehat{Y}, \tau, T): \mu \widehat{Y}=a F(\widehat{Y})\} .
\end{aligned}
$$

Denote $C\left(\mathbf{0}, \tau_{j}, 2 \pi / \omega_{0} \tau_{j}\right)$ the connected component of $\left(\mathbf{0}, \tau_{j}, 2 \pi / \omega_{0} \tau_{j}\right)$ in $\Sigma$, where $\omega_{0}=\sqrt{\left(a^{2}-\mu^{2}\right) /\left(1-b^{2}\right)}$ and $\tau_{j}(j=0,1,2, \ldots)$ are defined by Lemma 6 .

Lemma 8. Equation (44) has no periodic nonconstant solution when $\tau=0$.

Proof. Let $u(t)=y_{1}(t)+y_{2}(t)$. When $\tau=0$, we from (44) have

$$
\frac{d}{d t}[u(t)-b u(t-1)]=0
$$

Therefore, there exists $c_{1} \in \mathbb{R}$ such that

$$
u(t)=c_{1}+b u(t-1)
$$

Which implies that when $t \in[n, n+1]$, we have

$$
u(t)=c_{1} \sum_{i=0}^{n-1} b^{i}+b^{n} u(t-n)
$$

Hence we obtain $\lim _{t \rightarrow+\infty} u(t)=c_{1} /(1-b)$.

Let $v(t)=y_{1}(t)-y_{2}(t)$. With the similar process as above, it is obtained that there exists $c_{2} \in \mathbb{R}$ such that $\lim _{t \rightarrow+\infty} v(t)=$ $c_{2} /(1+b)$.

Then the conclusion follows from $\lim _{t \rightarrow+\infty} y_{1}(t)=$ $(1 / 2)\left[c_{1} /(1-b)+c_{2} /(1+b)\right]$, and the proof is complete.

Lemma 9. If $|b|<1 / 2$, then all periodic solution of (44) are uniformly bounded.

Proof. Let $Y(t)=\left(y_{1}(t), y_{2}(t)\right)^{T}$ be a periodic solution of (44) and $u(t)=y_{1}(t)+y_{2}(t)$. Then there exist $t_{1}$ and $t_{2}$ such that

$$
\begin{aligned}
& u\left(t_{1}\right)-b u\left(t_{1}-1\right)=\max _{t \in \mathbb{R}}[u(t)-b u(t-1)], \\
& u\left(t_{2}\right)-b u\left(t_{2}-1\right)=\min _{t \in \mathbb{R}}[u(t)-b u(t-1)]
\end{aligned}
$$

together with $T^{0}$ and $T_{0}$ such that

$$
u\left(T^{0}\right)=\max _{t \in \mathbb{R}^{+}} u(t), \quad u\left(T_{0}\right)=\min _{t \in \mathbb{R}^{+}} u(t),
$$

Now we take the case $-1 / 2<b<0$ as an example, and it has the same result when $0 \leqslant b<1 / 2$.
By (44), (48), and (H1), we have that

$$
u\left(t_{i}\right)=\frac{a}{u} \sum_{j=1}^{2} f\left(y_{j}\left(t_{i}-1\right)\right) \in\left[-\frac{2|a| L}{\mu}, \frac{2|a| L}{\mu}\right],
$$

$$
\begin{aligned}
u\left(t_{2}\right)-b u\left(t_{2}-1\right) & \leqslant u(t)-b u(t-1) \\
& \leqslant u\left(t_{1}\right)-b u\left(t_{1}-1\right)
\end{aligned}
$$

On one hand, we have

$$
\begin{aligned}
u(t) \leqslant & b u(t-1)+\left[u\left(t_{1}\right)-b u\left(t_{1}-1\right)\right] \\
\leqslant & b^{2} u(t-2)+b\left[u\left(t_{2}\right)-b u\left(t_{2}-1\right)\right] \\
& +\left[u\left(t_{1}\right)-b u\left(t_{1}-1\right)\right] \\
\leqslant & b^{2 m} u(t-2 m)+\sum_{i=1}^{m} b^{2 i-1}\left[u\left(t_{2}\right)-b u\left(t_{2}-1\right)\right] \\
& +\sum_{i=1}^{m} b^{2(i-1)}\left[u\left(t_{1}\right)-b u\left(t_{1}-1\right)\right] \\
\leqslant & \frac{b}{1-b^{2}}\left[u\left(t_{2}\right)-b u\left(t_{2}-1\right)\right] \\
& +\frac{1}{1-b^{2}}\left[u\left(t_{1}\right)-b u\left(t_{1}-1\right)\right] \\
\leqslant & \frac{2|a| L}{(1+b) \mu}-\frac{b^{2}}{1-b^{2}} u\left(T_{0}\right)-\frac{b}{1-b^{2}} u\left(T^{0}\right) .
\end{aligned}
$$

And on the other hand, we can obtain

$$
u(t) \geqslant-\frac{2|a| L}{(1+b) \mu}-\frac{b}{1-b^{2}} u\left(T_{0}\right)-\frac{b^{2}}{1-b^{2}} u\left(T^{0}\right) .
$$

It follows that

$$
\begin{gathered}
u\left(T_{0}\right) \geqslant \frac{1-b^{2}}{1+b-b^{2}}\left[-\frac{2|a| L}{(1+b) \mu}-\frac{b^{2}}{1-b^{2}} u\left(T^{0}\right)\right], \\
u\left(T^{0}\right) \leqslant \frac{2|a| L}{(1+2 b) \mu} .
\end{gathered}
$$

Similarly, one can prove that

$$
u\left(T_{0}\right) \geqslant-\frac{2|a| L}{(1+2 b) \mu} .
$$

Which implies that

$$
-\frac{2|a| L}{(1+2 b) \mu} \leqslant u(t) \leqslant \frac{2|a| L}{(1+2 b) \mu} .
$$

Let $v(t)=y_{1}(t)-y_{2}(t)$. In the same way, it can be verified that

$$
-\frac{2|a| L}{(1+2 b) \mu} \leqslant v(t) \leqslant \frac{2|a| L}{(1+2 b) \mu} .
$$

Then $y_{1}(t)$ and $y_{2}(t)$ are uniformly bounded, and the proof is complete. 


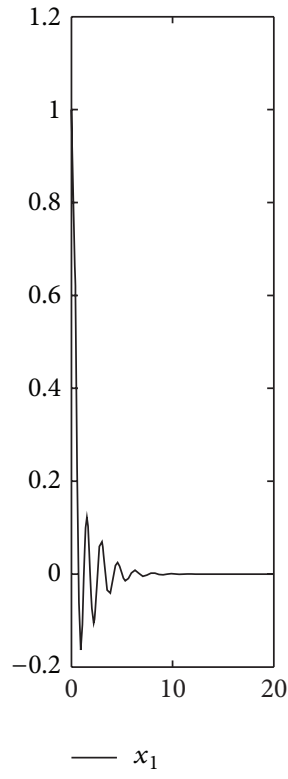

(a)

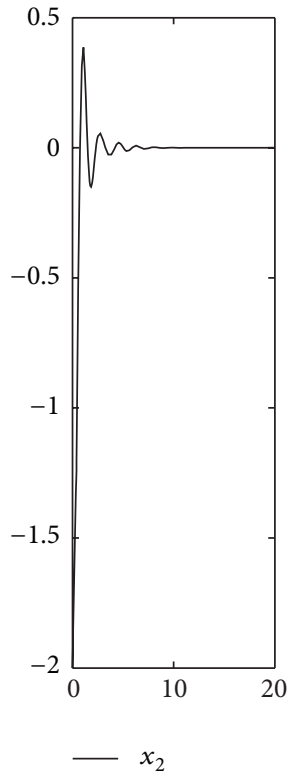

(b)

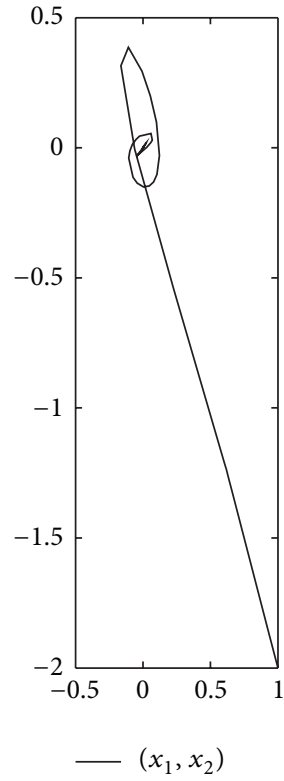

(c)

FIGURE 1: For system (1), the equilibrium $(0,0)$ is asymptotically stable when $\tau=0.55<\tau_{0} \approx 0.6378$.

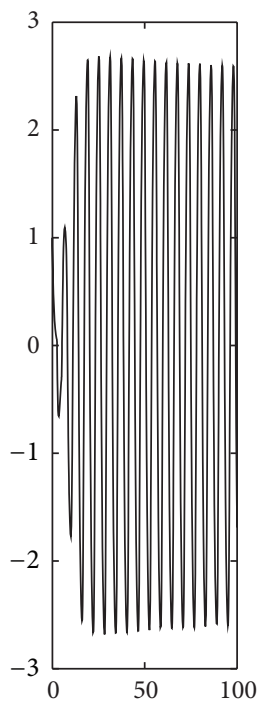

(a)

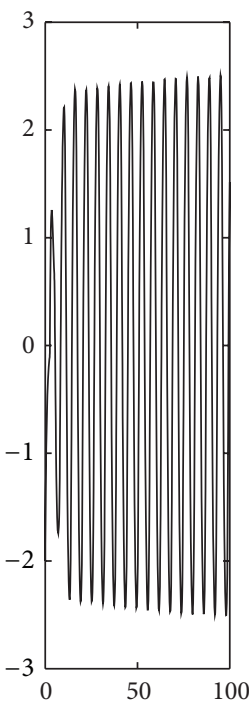

(b)

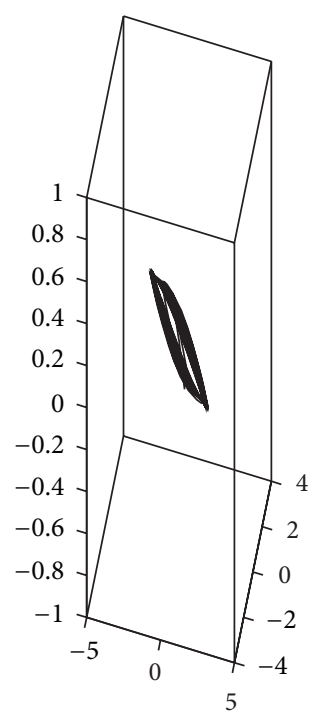

(c)

Figure 2: The system (1) undergoes a Hopf bifurcation at $(0,0)$, and the bifurcating periodic solution is asymptotically stable when $\tau=2.5 \epsilon$ $\left(\tau_{0}, \tau_{1}\right)$.

Lemma 10. If $a<-\mu$ or $a>\mu / M$, then (44) has no periodic nonconstant solution of period 1 , where $M=$ $\min \left\{f^{\prime}(-2|a| L /(1+2 b) \mu), f^{\prime}(2|a| L /(1+2 b) \mu)\right\}$.

Proof. Let $\left(y_{1}(t), y_{2}(t)\right)$ be a periodic solution to (44) of period 1 . Then it is a periodic solution to the following system of ordinary differential equations:

$$
\begin{aligned}
& \dot{u}_{1}(t)-b \dot{u}_{2}(t)=-\mu \tau u_{1}(t)+a \tau f\left(u_{1}(t)\right), \\
& \dot{u}_{2}(t)-b \dot{u}_{1}(t)=-\mu \tau u_{2}(t)+a \tau f\left(u_{2}(t)\right),
\end{aligned}
$$

where $\cdot$ denotes $d / d t$. Denote

$$
G=\left\{u \in \mathbb{R}^{2}: u_{j} \in\left[-\frac{2|a| L}{(1+2 b) \mu}, \frac{2|a| L}{(1+2 b) \mu}\right], j=1,2\right\} \text {. }
$$




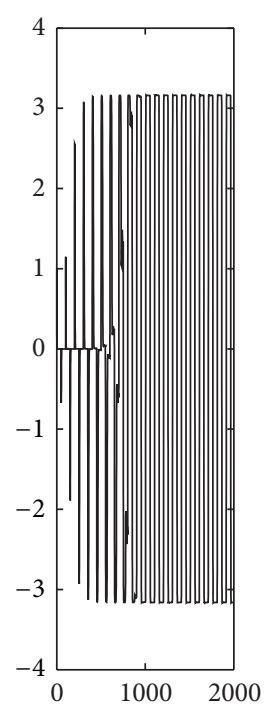

(a)

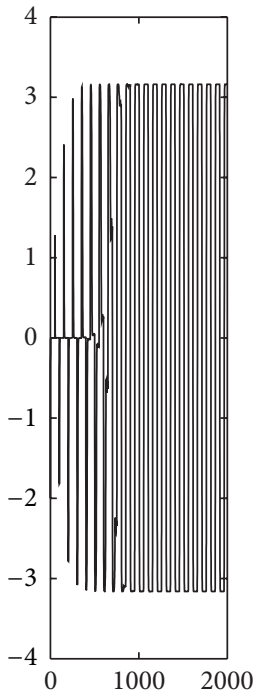

(b)

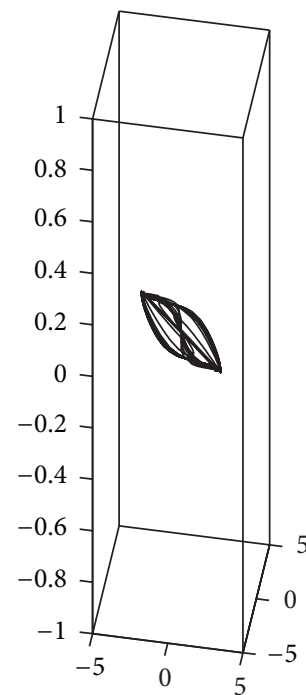

(c)

FIGURE 3: The system (1) undergoes a Hopf bifurcation at $(0,0)$ with sufficiently large $\tau=50$.

Lemma 9 shows that the periodic solution of (44) belong to the region $G$. Clearly, (57) is equivalent to

$$
\begin{aligned}
\dot{u}_{1} & =\frac{\tau}{1-b^{2}}\left[-\mu\left(u_{1}+b u_{2}\right)+a\left(f\left(u_{1}\right)+b f\left(u_{2}\right)\right)\right] \\
& \stackrel{\text { def }}{=} P\left(u_{1}, u_{2}\right), \\
\dot{u}_{2} & =\frac{\tau}{1-b^{2}}\left[-\mu\left(u_{2}+b u_{1}\right)+a\left(f\left(u_{2}\right)+b f\left(u_{1}\right)\right)\right] \\
& \stackrel{\text { def }}{=} Q\left(u_{1}, u_{2}\right),
\end{aligned}
$$

Then

$$
\frac{\partial P}{\partial u_{1}}+\frac{\partial Q}{\partial u_{2}}=\frac{\tau}{1-b^{2}}\left[-2 \mu+a\left(f^{\prime}\left(u_{1}\right)+f^{\prime}\left(u_{2}\right)\right)\right]
$$

From (H2), it is easy to see that $\partial P / \partial u_{1}+\partial Q / \partial u_{2}<0$ when $a<-\mu$ and $\partial P / \partial u_{1}+\partial Q / \partial u_{2}>0$ when $a>\mu / M$. Thus, the classical Bendixson's criterion implies that (57) has no nonconstant periodic solution in the region $G$, and the proof is complete.

Up to now, we have prepared sufficiently to state the following global Hopf bifurcation results.

Theorem 11. If $|b|<1 / 2$ and either $a<-\mu$ or $a>\mu / M$ holds, then (44) has at least one periodic solution for $\tau>\tau_{j}(j \geqslant 1)$.

Proof. First note that the stationary points $\left(\mathbf{0}, \tau_{j}, 2 \pi / \omega_{0} \tau_{j}\right)$ of (44) are nonsingular and isolate centers (see [14]) under the assumption $|a|>\mu$ for each $\tau>\tau_{j}(j \geqslant 1)$. By (25), there exist $\epsilon>0, \delta>0$, and a smooth curve $\lambda:\left(\tau_{j}-\delta, \tau_{j}+\delta\right) \rightarrow \mathbb{C}$, such that

$$
\begin{gathered}
\Delta(\lambda(\tau))=\Delta_{(\mathbf{0}, \tau, T)}(\lambda(\tau))=0, \\
\left|\lambda(\tau)-i \omega_{0} \tau_{j}\right|<\epsilon,
\end{gathered}
$$

for all $\tau \in\left[\tau_{j}-\delta, \tau_{j}+\delta\right]$, where $\Delta$ is defined as (23), and

$$
\lambda\left(\tau_{j}\right)=i \omega_{0} \tau_{j},\left.\quad \frac{d \operatorname{Re}(\lambda(\tau))}{d \tau}\right|_{\tau=\tau_{j}}>0
$$

Let

$$
\Omega_{\epsilon}=\left\{(u, p): 0<u<\epsilon,\left|p-\frac{2 \pi}{\omega_{0} \tau_{j}}\right|<\epsilon\right\} .
$$

Clearly, if $\tau-\tau_{j} \leqslant \delta$ and $(u, p) \in \Omega_{\epsilon}$ such that $\Delta_{(0, \tau, T)}(u+$ $i 2 \pi / p)=0$, then $\tau=\tau_{j}, u=0$, and $p=2 \pi / \omega_{0} \tau_{j}$. Moreover, if we put

$$
H^{ \pm}\left(0, \tau_{j}, \frac{2 \pi}{\omega_{0} \tau_{j}}\right)(u, p)=\Delta_{(0, \tau \pm \delta, T)}\left(u+\frac{i 2 \pi}{p}\right),
$$

at $m=1$, we have, from $\operatorname{Re} \lambda^{\prime}\left(\tau_{j}\right)>0$, that the crossing number is

$$
\begin{aligned}
\gamma_{1}\left(0, \tau_{j}, \frac{2 \pi}{\omega_{0} \tau_{j}}\right)= & \operatorname{deg}_{B}\left(H^{-}\left(0, \tau_{j}, \frac{2 \pi}{\omega_{0} \tau_{j}}\right), \Omega_{\epsilon}\right) \\
& -\operatorname{deg}_{B}\left(H^{+}\left(0, \tau_{j}, \frac{2 \pi}{\omega_{0} \tau_{j}}\right), \Omega_{\epsilon}\right)=-1 .
\end{aligned}
$$

Using the local Hopf bifurcation theorem for NFDE [14, Theorem 5.6], we conclude that the connected component 
$C\left(\mathbf{0}, \tau_{j}, 2 \pi / \omega_{0} \tau_{j}\right)$ through $\left(\mathbf{0}, \tau_{j}, 2 \pi / \omega_{0} \tau_{j}\right)$ in $\Sigma$ is nonempty, and thus $C\left(\mathbf{0}, \tau_{j}, 2 \pi / \omega_{0} \tau_{j}\right)$ is unbounded by the global Hopf bifurcation theorem given by Krawcewicz et al. [14, Theorem 5.14].

Lemma 9 implies that the projection of $C\left(\mathbf{0}, \tau_{j}, 2 \pi / \omega_{0} \tau_{j}\right)$ onto the $Y$-space is bounded. Meanwhile, the projection of $C\left(\mathbf{0}, \tau_{j}, 2 \pi / \omega_{0} \tau_{j}\right)$ onto $\tau$-space is bounded below from Lemma 8.

By the definition of $\tau_{j}$, we know that

$$
2 \pi<\omega_{0} \tau_{j}<2(j+1) \pi, \quad j \geqslant 1,
$$

under the assumptions that $|a|>\mu$ and $|b|<1$, which implies

$$
\frac{1}{j+1}<\frac{2 \pi}{\omega_{0} \tau_{j}}<1 .
$$

Applying Lemma 10, one has $1 /(j+1)<T<1$ if $(Y, \tau, T) \in C\left(\mathbf{0}, \tau_{j}, 2 \pi / \omega_{0} \tau_{j}\right)$ for $j \geqslant 1$, when $|b|<1 / 2$ and either $a<-\mu$ or $a>\mu / M$ holds. Thus in order for $C\left(\mathbf{0}, \tau_{j}, 2 \pi / \omega_{0} \tau_{j}\right)$ to be unbounded, its projection onto the $\tau$ space must be unbounded. Consequently, the projection of $C\left(\mathbf{0}, \tau_{j}, 2 \pi / \omega_{0} \tau_{j}\right)$ onto the $\tau$-space include $\left[\tau_{j}, \infty\right)$ for $j \geqslant 1$ when $|b|<1 / 2$ and either $a<-\mu$ or $a>\mu / M$ holds. The proof is complete.

Next we carry out some numerical simulations for (1).

Let $f(x)=\arctan (x)$, and assume that $\mu=1.2, a=-3$, and $b=0.2$. From Lemma 4 , it is obtained that $\omega_{0} \approx 2.8062$ and $\tau_{0} \approx 0.6378$. Accordingly, it is known that $(0,0)$ is asymptotically stable for $\tau \in\left(0, \tau_{0}\right)$ and unstable for $\tau>$ $\tau_{0}$, and Hopf bifurcation at the origin occurs when $\tau=\tau_{0}$ by Theorem 5 . From Theorem 7, the direction of the Hopf bifurcation at $\tau=\tau_{0}$ is supercritical, and the bifurcating periodic solutions are asymptotically stable. Furthermore, according to Theorem 11, (1) with this set of parameters has at least one periodic solution when $\tau>\tau_{j}(j \geqslant 1)$. The corresponding numerical simulation results are shown in Figures 1, 2, and 3.

\section{Acknowledgment}

This work is supported by the Fundamental Research Funds for the Central Universities, (no. DL11AB02).

\section{References}

[1] J. J. Wei and S. G. Ruan, "Stability and global Hopf bifurcation for neutral differential equations," Acta Mathematica Sinica, vol. 45, no. 1, pp. 93-104, 2002.

[2] C. Wang and J. Wei, "Hopf bifurcation for neutral functional differential equations," Nonlinear Analysis: Real World Applications, vol. 11, no. 3, pp. 1269-1277, 2010.

[3] Y. Qu, M. Y. Li, and J. Wei, "Bifurcation analysis in a neutral differential equation," Journal of Mathematical Analysis and Applications, vol. 378, no. 2, pp. 387-402, 2011.

[4] Y. Chen and J. Wu, "Slowly oscillating periodic solutions for a delayed frustrated network of two neurons," Journal of Mathematical Analysis and Applications, vol. 259, no. 1, pp. 188208, 2001.
[5] J. Wei and S. Ruan, "Stability and bifurcation in a neural network model with two delays," Physica D, vol. 130, no. 3-4, pp. 255-272, 1999.

[6] T. Faria, "On a planar system modelling a neuron network with memory," Journal of Differential Equations, vol. 168, no. 1, pp. 129-149, 2000.

[7] J. Wei, M. G. Velarde, and V. A. Makarov, "Oscillatory phenomena and stability of periodic solutions in a simple neural network with delay," Nonlinear Phenomena in Complex Systems, vol. 5, no. 4, pp. 407-417, 2002.

[8] J. J. Wei, C. R. Zhang, and X. L. Li, "Bifurcation in a twodimensional neural network model with delay," Applied Mathematics and Mechanics, vol. 26, no. 2, pp. 193-200, 2005.

[9] J. K. Hale and M. V. Lunel, Introduction to FunctionalDifferential Equations, vol. 99 of Applied Mathematical Sciences, Springer, New York, NY, USA, 1993.

[10] C. Wang and J. Wei, "Normal forms for NFDEs with parameters and application to the lossless transmission line," Nonlinear Dynamics, vol. 52, no. 3, pp. 199-206, 2008.

[11] S. Wiggins, Introduction to Applied Nonlinear Dynamical Systems and Chaos, vol. 2 of Texts in Applied Mathematics, Springer, New York, NY, USA, 1990.

[12] B. D. Hassard, N. D. Kazarinoff, and Y. H. Wan, Theory and Applications of Hopf Bifurcation, vol. 41 of London Mathematical Society Lecture Note Series, Cambridge University Press, Cambridge, Mass, USA, 1981.

[13] J. Carr, Applications of Centre Manifold Theory, vol. 35 of Applied Mathematical Sciences, Springer, New York, NY, USA, 1981.

[14] W. Krawcewicz, J. Wu, and H. Xia, "Global Hopf bifurcation theory for condensing fields and neutral equations with applications to lossless transmission problems," The Canadian Applied Mathematics Quarterly, vol. 1, no. 2, pp. 167-220, 1993.

[15] J. H. Wu, "Global continua of periodic solutions to some difference-differential equations of neutral type," The Tohoku Mathematical Journal, vol. 45, no. 1, pp. 67-88, 1993.

[16] J. Wu and H. Xia, "Self-sustained oscillations in a ring array of coupled lossless transmission lines," Journal of Differential Equations, vol. 124, no. 1, pp. 247-278, 1996.

[17] M. Weedermann, "Hopf bifurcation calculations for scalar neutral delay differential equations," Nonlinearity, vol. 19, no. 9, pp. 2091-2102, 2006. 


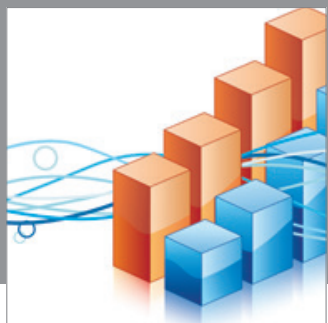

Advances in

Operations Research

mansans

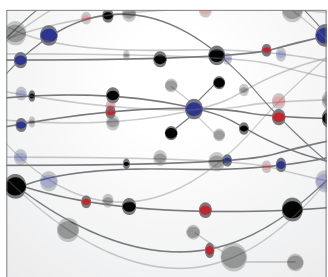

The Scientific World Journal
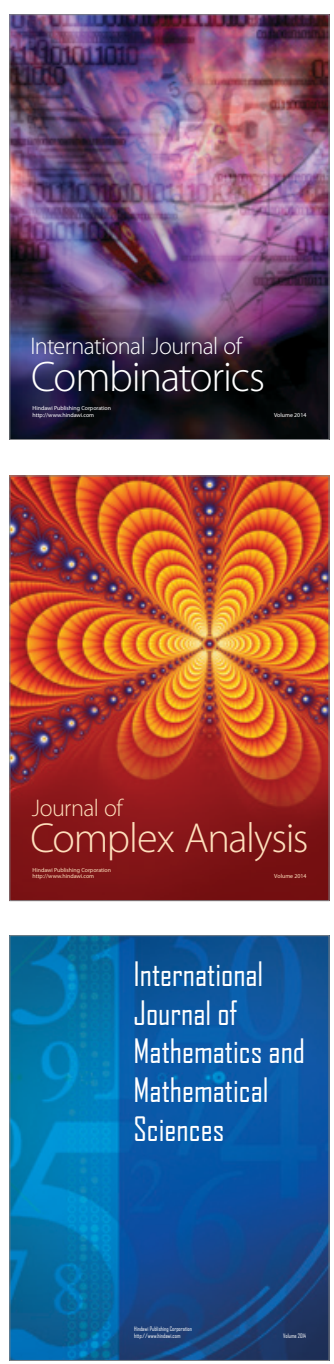
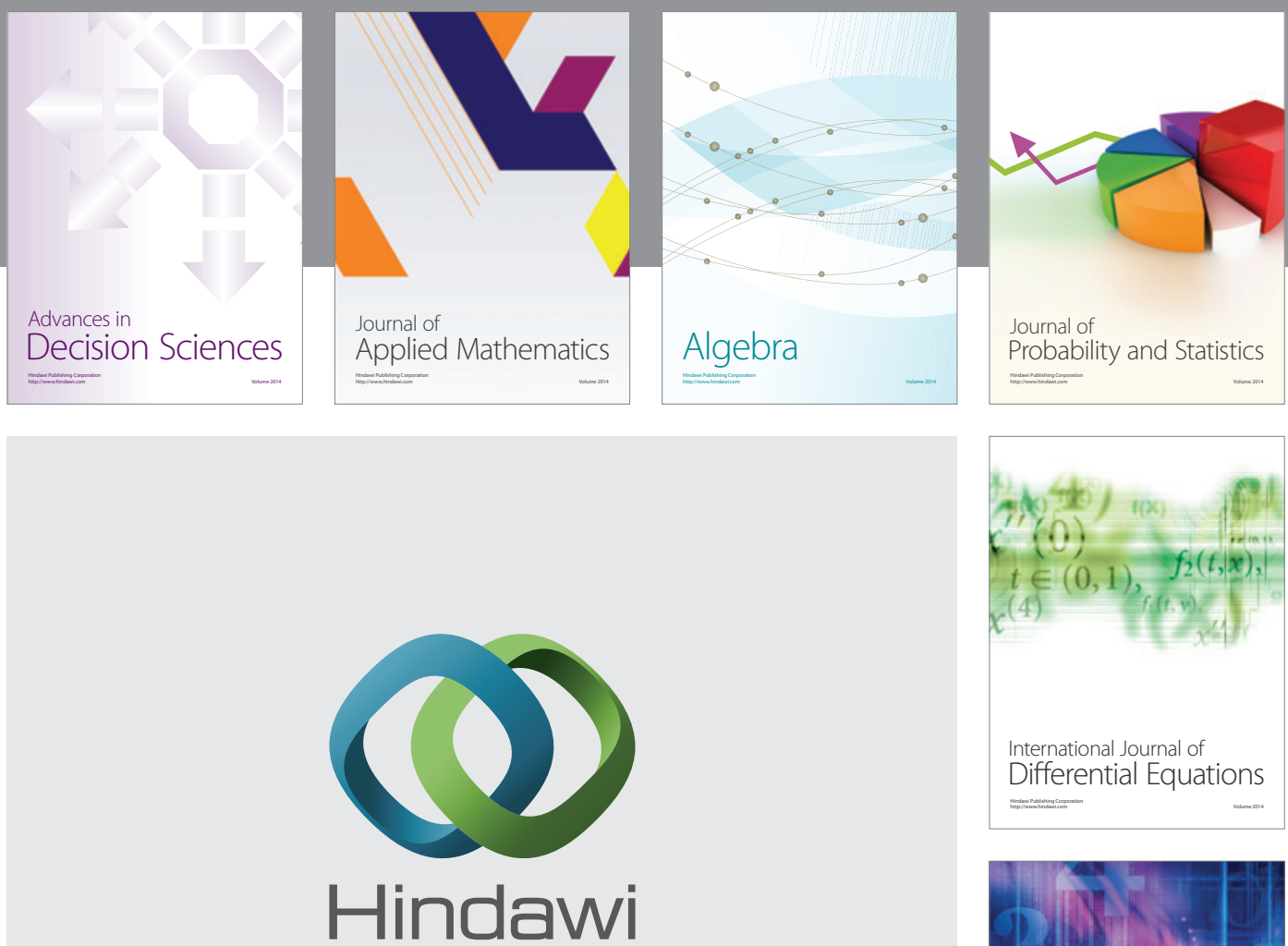

Submit your manuscripts at http://www.hindawi.com
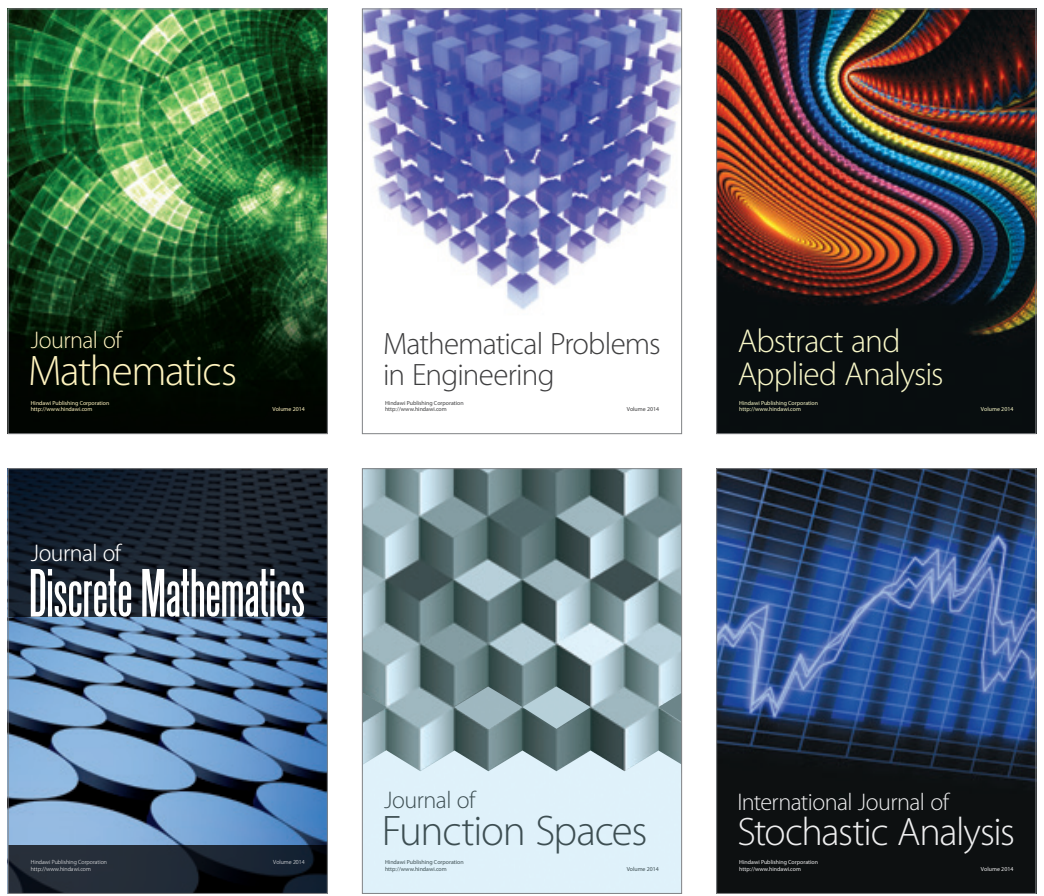

Journal of

Function Spaces

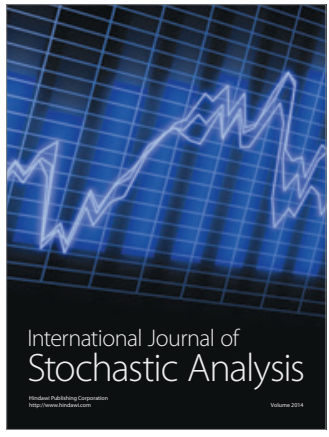

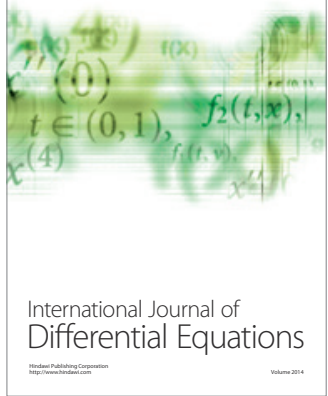
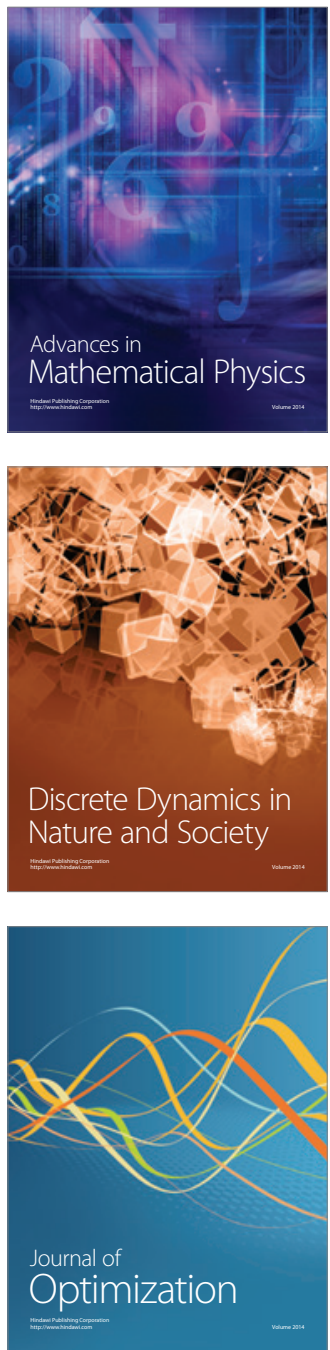